\title{
The overnight smackdown: avoiding on-call arguments
}

Andy, the general surgery resident oncall, has had an exhausting night. His head finally touches the pillow at 4 am. At 4:10 am he's paged. When he returns the page, it is clear from his tone of voice that he's annoyed. It's Mark, the senior internal medicine resident, requesting an urgent consult on an 83year-old man to rule out ischemic bowel. The patient was admitted several days before for an acute chronic obstructive pulmonary disease exacerbation; however, he developed vague abdominal pain and a borderline lactate within the previous 6 hours. Andy wants to wait until the morning or until a computed tomographic (CT) scan is performed. The CT was declined by radiology and Mark doesn't want to wait until morning for a consult. The argument begins and quickly escalates.

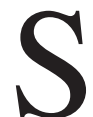

ituations similar to this fictional vignette play out repeatedly most nights during on-call at academic institutions. The cause of many (not all) of these disputes is simple: residents, who essentially run the show while on-call overnight, tend to have less confidence and knowledge, and therefore a lower threshold for ordering exams and requesting consults. Their rationale is simple: err on the side of caution. And it is easy to see their point. There has been evidence, for example, that mortality among patients admitted on weekends, (also a period when more junior physicians tend to be in charge $\left.{ }^{1,2}\right)$, is greater than among patients admitted on weekdays. ${ }^{3}$

As a fourth-year resident in radiology, I'd like to offer some suggestions on how to avoid these late-night arguments:

1. Don't answer pages with hostility and avoid walling off before you've heard the story. Many people seem to do this, perhaps in the hope that it will prevent future consults. Starting off in a cordial, friendly and professional manner will set the tone for the consult, and the subse-

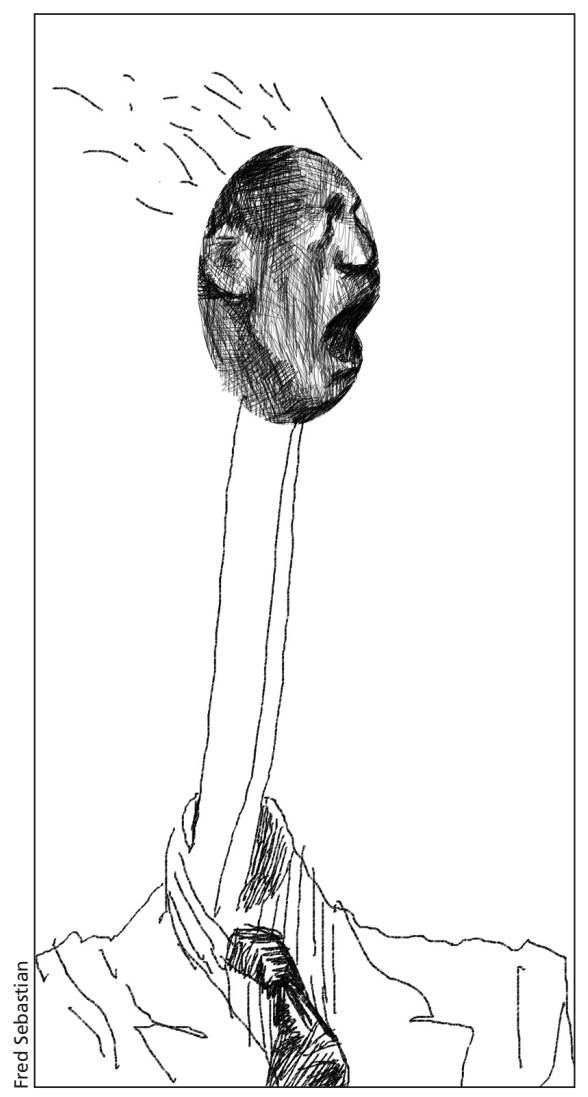

quent discussion will likely go more smoothly.

2. If you are skeptical about whether the requested exam or consult is urgent and must be performed overnight, clarify the request and listen to the whole story. You may have misunderstood the request. If you remain skeptical and believe it should wait until the morning, explain your rationale to the referring colleague in a cordial manner. Should this fail, call your fellow or staff for advice. This is a much better solution than starting a war over the telephone.

3. If you do end up in a serious argument, avoid the temptation to send a nasty email when your on-call ends. Talk to someone in your own department. Support can be comforting and if they believe you were in fact wrong then you may avoid further escalation by apologizing to the person.

4. If you believe you were unfairly harassed or intimidated by another service (student, resident, fellow, or staff), remember that this is unacceptable and no longer tolerated. Universities and hospitals have harassment and intimidation officers who are there to help you.

Probably the single best tip I've learned is to remember that the person on the line has not called simply to wake you up out of spite. They have a patient they are worried about and need help. Remember that residents and medical students are often unsure about some aspect of a particular case. Oncall overnight, there are not that many others to turn to. If you are called, someone thinks you can help.

As a resident in radiology, we have to rotate through many of the medical and surgical subspecialties during our intern (first) year. The 2 constants that I learned during my intern year about these on-call arguments are:

There are 2 sides to every argument. Everyone thinks that they're right.

\section{Mark Otto Baerlocher}

Post-graduate year 4

Radiology residency training program

University of Toronto

Toronto, Ont.

\section{REFERENCES}

1. Thorpe KE. House staff supervision and working hours: implications of regulatory change in New York State. JAMA 1990;263:3177-81.

2. McKee M, Black N. Does the current use of junior doctors in the United Kingdom affect the quality of medical care? Soc Sci Med 1992;34:549-58.

3. Bell CM, Redelmeier DA. Mortality among patients admitted to hospitals on weekends as compared with weekdays. $N$ Engl J Med 2001;345: 663-8.

Have you got an opinion about this article? Post your views at www.cmaj.ca. Potential Salon contributors are welcome to send a query to salon@cma.ca. 